\title{
MULTIPLE POINTS OF TRANSIENT RANDOM WALKS
}

\author{
JOEL H. PITT
}

\begin{abstract}
We determine the asymptotic behavior of the expected numbers of points visited exactly $j$ times and at least $j$ times in the first $\boldsymbol{n}$ steps of a transient random walk on a discrete Abelian group. We prove that the strong law of large numbers holds for these multiple point ranges.
\end{abstract}

Let $X_{1}, X_{2}, \cdots$ be a sequence of independent identically distributed random variables (taking values in an arbitrary countable Abelian group), and let $S_{1}=X_{1}, S_{2}=X_{1}+X_{2}, S_{3}=X_{1}+X_{2}+X_{3}+\cdots$ be the sequence of partial sums of $\left\{X_{n}\right\}$. The range $R_{n}$ of the random walk associated with $X_{1}, X_{2}, \cdots$ is the number of distinct values assumed by the finite sequence of partial sums $S_{1}, S_{2}, \cdots, S_{n}$. Dvoretsky and Erdös [1] (and by different means Spitzer, Kesten and Whitman [3, pp. 38-40]) have shown that for any random walk $\lim _{n \rightarrow \infty} E\left(R_{n} / n\right)=1-F$, where $F$ is the probability that $\exists n \ni S_{n}=0$, and, in fact, the strong law of large numbers applies to the range in the sense that $\lim _{n \rightarrow \infty} R_{n} / n=1-F$ a.s. (The latter result is of interest principally when the walk is transient, i.e. $F \neq 1$; Dvoretsky and Erdös obtain the stronger $\lim _{n \rightarrow \infty} R_{n} / E\left(R_{n}\right)=1$ a.s. for simple random walk in the plane.)

We obtain analogous results concerning the number of distinct values assumed exactly $j$ times and at least $j$ times by the sequence $S_{1}, S_{2}, \cdots, S_{n}$.

We introduce the notation:

$$
\begin{aligned}
R_{n}^{j}= & \text { the \# of distinct values occurring at least } j \text { times in } \\
& \text { the sequence } S_{1}, \cdots, S_{n} . \\
R_{n}^{(j)}= & \text { the \# of distinct values occurring exactly } j \text { times in } \\
& \text { the sequence } S_{1}, \cdots, S_{n} .
\end{aligned}
$$

Our main result is:

THEOREM. For any random walk and any $j, \lim _{n \rightarrow \infty}\left(R_{n}^{j} / n\right)=(1-F) F^{j-1}$ a.s., and $\lim _{n \rightarrow \infty}\left(R_{n}^{(j)} / n\right)=(1-F)^{2} F^{j-1}$ a.s.

Received by the editors January 29, 1973.

AMS (MOS) subject classifications (1970). Primary 60F15, 60J15; Secondary 28A65, 60G50.

Key words and phrases. Range of random walk, strong law of large numbers, ergodic theorem.

(c) American Mathematical Society 1974 
Note. (1) For recurrent walks this result is a direct consequence of the result for $j=1$, and of no interest.

(2) The corresponding result for simple walk on $Z^{n}, n \geqq 1$, is stated by Erdös and Taylor [2]. Their method is entirely different and applies only to walks on $Z^{n}$.

Proof. The proof is inductive. The result is already known for $R_{n}^{1}$; so all we need do is establish the induction step: $R_{n}^{j} / n \rightarrow(1-F) F^{j-1}$ a.s. implies $R_{n}^{(j)} / n \rightarrow\left(1-F^{2}\right) F^{j-1}$ a.s. and $R_{n}^{j+1} / n \rightarrow(1-F) F^{j-1}$ a.s.

We assume then that $R_{n}^{j} / n \rightarrow(1-F) F^{j-1}$ a.s. and turn to the first order of business, the determination of the behavior of $E\left(R_{n}^{(j)}\right) / n$ and $E\left(R_{n}^{j+1}\right) / n$ as $n \rightarrow \infty$. To this end we write:

$$
\begin{aligned}
Z_{k} & =1, \quad \text { if there are exactly } j-1 \text { indices } l<k \ni S_{l}=S_{k}, \\
& =0, \quad \text { otherwise. } \\
Y_{k, n} & =1, \quad \text { if there are exactly } j-1 \text { indices } l<k \text { such that } \\
& \quad S_{l}=S_{k} \text { and } S_{k+1} \neq S_{k}, S_{k+2} \neq S_{k}, \cdots, S_{n} \neq S_{k}, \\
& =0, \quad \text { otherwise. }
\end{aligned}
$$

We have $R_{n}^{(j)}=\sum_{k=1}^{n} Y_{k, n}$ so that $E\left(R_{n}^{(j)}\right)=\sum_{k=1}^{n} E\left(Y_{k, n}\right)$. Also, $X_{k+1}, \cdots$, $X_{n}$, are independent of $Z_{k}$, hence

$$
\begin{aligned}
E\left(Y_{k, n}\right) & =E\left(Z_{k}\right) P\left(X_{k+1} \neq 0, X_{k+1}+X_{k+2} \neq 0, \cdots, X_{k+1}+\cdots+X_{k+n} \neq 0\right) \\
& =E\left(Z_{k}\right) P\left(S_{1} \neq 0, \cdots, S_{n-k} \neq 0\right) .
\end{aligned}
$$

But also $R_{n}^{j}=\sum_{k=1}^{n} Z_{k}$ and, in view of the induction hypothesis and the Lebesgue dominated convergence theorem,

$$
\sum_{k=1}^{n} E\left(Z_{k}\right) / n=E\left(R_{n}^{j}\right) / n \rightarrow(1-F) F^{j-1} .
$$

Furthermore, if we write, $f_{k}=P\left(S_{1} \neq 0, S_{2} \neq 0, \cdots, S_{k-1} \neq 0, S_{k}=0\right)$ we have $P\left(S_{1} \neq 0, \cdots, S_{n-k} \neq 0\right)=1-\sum_{i=1}^{n-k} f_{i}$ and, as $n \rightarrow \infty$,

$$
1-\sum_{i=1}^{n-k} f_{i} \rightarrow 1-F .
$$

A straightforward argument then yields

$$
E\left(R_{n}^{(j)}\right) / n \rightarrow(1-F)^{2} F^{j-1},
$$

and the further observation that $R_{n}^{j+1}=R_{n}^{j}-R_{n}^{(j)}$ gives

$$
E\left(R_{n}^{j+1}\right) / n \rightarrow(1-F) F^{j} .
$$


To obtain the desired strong laws of large numbers we introduce the new sequences of random variables defined for $n=1,2, \cdots, k=1,2, \cdots$ :

$T_{k, n}^{(j)}=$ the number of distinct values occurring exactly $j$ times in $S_{(k-1) n+1}, \cdots, S_{k n}$,

$T_{k, n}^{j+1}=$ the number of distinct values occurring at least $j+1$ times in $S_{(k-1) n+1}, \cdots, S_{k n}$.

For any $m$ we have

$$
\begin{aligned}
& R_{m n}^{(j)} \leqq \sum_{k=1}^{m} T_{k, n}^{(j)}+E_{m, n}, \\
& R_{m n}^{j+1} \leqq \sum_{k=1}^{m} T_{k, n}^{j+1}+E_{m, n},
\end{aligned}
$$

where $E_{m, n}=$ the number of distinct sums which occur in at least two different blocks

$$
\left\{S_{(k-1) n+1}, \cdots, \quad S_{k n}\right\}, \quad\left\{S_{\left(k^{\prime}-1\right) n+1}, \cdots, S_{k^{\prime} n}\right\}, \quad k, k^{\prime} \leqq m .
$$

We prove below that

$$
\limsup _{n \rightarrow \infty} \limsup _{m \rightarrow \infty} E_{m, n} / m n=0,
$$

and complete our proof of the theorem assuming the truth of this lemma.

We let $m \rightarrow \infty$ in (5) and obtain

$$
\limsup _{m \rightarrow \infty} \frac{R_{m n}^{(j)}}{m n} \leqq \lim _{m \rightarrow \infty} \frac{\sum_{k=1}^{m} T_{k, n}^{(j)}}{m n}+\lim _{m \rightarrow \infty} \frac{E_{m, n}}{m n} .
$$

By the strong law of large numbers applied to the sequence of (bounded) independent identically distributed random variables $T_{k, n}, k=1,2, \cdots$,

$$
\lim _{m \rightarrow \infty} \frac{\sum_{k=1}^{m} T_{k, n}^{(j)}}{m n}=\frac{1}{n} \lim _{m \rightarrow \infty} \frac{\sum_{k=1}^{m} T_{k, n}^{(j)}}{m}=\frac{E\left(T_{1, n}^{(j)}\right)}{n}=\frac{E\left(R_{n}^{(j)}\right)}{n} .
$$

(7) yields a bound on $\lim \sup R_{k}^{(j)} / k$ as $k \rightarrow \infty$ through a subsequence consisting of multiples of $n$. The restriction of the mode of approach to a subsequence may be eliminated by observing that

$$
\begin{aligned}
R_{k}^{(j)} / k & \leqq \frac{R_{[k / n]_{n}+\left(k-[k / n]_{n}\right)}^{(j)} \leqq R_{[k / n]_{n}}^{(j)}\left[[k / n]_{n}+1 /[k / n]\right.}{[k / n]_{n}} \\
& =R_{m n}^{(j)} / m n+o(1)
\end{aligned}
$$

so that lim $\sup _{k \rightarrow \infty} R_{k}^{(j)} / k \leqq \lim \sup _{m \rightarrow \infty} R_{m n}^{(j)} / m n$ for any $n$. 
Substituting (8) in (7), letting $n \rightarrow \infty$ and using (3) yields

$$
\limsup _{k \rightarrow \infty} R_{k}^{(j)} / k \leqq(1-F)^{2} F^{j-1} \text { a.s. }
$$

Trivial adjustments in the argument which led from (5) to (9) allow us to use (6) to conclude

$$
\limsup _{k \rightarrow \infty} R_{k}^{j+1} / k \leqq(1-F) F^{j}
$$

But now we complete our argument with ease. For,

$$
\liminf _{k \rightarrow \infty} R_{k}^{(j)} / k \geqq \liminf _{k \rightarrow \infty} R_{k}^{j} / k-\lim \sup R_{k}^{j+1} / k,
$$

and in view of our induction hypothesis and (10) the right side is $\geqq$ $(1-F)^{2} F^{j-1}$. Finally

$$
\lim _{k \rightarrow \infty} R_{k}^{j+1} / k=\lim _{k \rightarrow \infty} R_{k}^{j} / k-\lim _{k \rightarrow \infty} R_{k}^{(j)} / k=(1-F) F^{j} .
$$

Proof of Lemma. We define the random variable $\xi_{i}^{n}$ by

$$
\begin{aligned}
& \xi_{i}^{n}=1, \quad \text { if } S_{i} \neq S_{i+1}, S_{i} \neq S_{i+2}, \cdots, S_{i} \neq S_{([i / n]+1)_{n}} \\
& \text { but } \exists k>i \ni S_{k}=S_{i} \text { or } n \mid i \text { and } \exists k>i \ni S_{k}=S_{i} \text {, } \\
& =0 \text {, otherwise. }
\end{aligned}
$$

Clearly $0 \leqq E_{m, n} \leqq \sum_{i=1}^{m n} \xi_{i}^{n}$ (in fact, $E_{m, n} \leqq \sum_{i=1}^{(m-1) n} \xi_{i}^{n}$ ). We now choose an $n^{\prime}$ corresponding to $n$ so that $n^{\prime} \rightarrow \infty$ as $n \rightarrow \infty$ and $n^{\prime} \mid n \rightarrow 0$. We let

$$
\begin{aligned}
\eta_{i}^{n}=1, & \text { if } S_{i} \neq S_{i+1}, S_{i} \neq S_{i+2}, \cdots, S_{i} \neq S_{i+n^{\prime}} \\
& \quad \text { but } \exists k>i+n^{\prime} \ni S_{k}=S_{i}, \\
& =0, \text { otherwise. }
\end{aligned}
$$

The sequence of random variables $\eta_{i}^{n}$ is stationary ergodic, hence

$$
\lim _{k \rightarrow \infty} \frac{1}{k} \sum_{i=1}^{k} \eta_{i}^{n}=E\left(\eta_{i}^{n}\right)=\sum_{n^{\prime}}^{\infty} f_{j}
$$

by the ergodic theorem. Also $\xi_{i}^{n} \leqq \eta_{i}^{n}$ unless $n \mid i$ or $[i / n]+n-i<n^{\prime}$, so

Hence

$$
0 \leqq E_{m, n} \leqq \sum_{i=1}^{m n} \xi_{i}^{n}+m \cdot n^{\prime}
$$

$$
0 \leqq \limsup _{m \rightarrow \infty} \frac{E_{m, n}}{m n} \leqq \lim _{m \rightarrow \infty} \frac{\sum_{i=1}^{m n} \xi_{i}^{n}}{m n}+\frac{n^{\prime}}{n}=\sum_{n^{\prime}}^{\infty} f_{j}+\frac{n^{\prime}}{n} .
$$

And as $n \rightarrow \infty, \sum_{n}^{\infty} f_{j}+n^{\prime} / n \rightarrow 0$ by our choice of $n^{\prime}$. 


\section{BIBLIOGRAPHY}

1. A. Dvoretsky and P. Erdös, Some problems on random walk in space, Proc. Second Berkeley Sympos. on Math. Statist. and Probability, Univ. of California Press, Berkeley and Los Angeles, 1951, pp. 353-368. MR 13, 852.

2. P. Erdös and S. J. Taylor, Some problems concerning the structure of random walk paths, Acta Math. Acad. Sci. Hungar. 11 (1960), 137-162. MR 22 \#12599.

3. F. Spitzer, Principles of random walk, University Series in Higher Math., Van Nostrand, Princeton, N.J., 1964. MR 30 \#1521.

Department of Mathematics, State University College at New Paltz, New PALTZ, NeW YORK 12561 